YCTP-P-28-99

hep-ph/yymmmnn

\title{
Enhanced Global Symmetries and a Strong Electroweak Sector
}

\author{
Francesco SANNINO [0 \\ Department of Physics, Yale University, New Haven, \\ CT 06520-8120, USA.
}

\begin{abstract}
In this talk I review the intriguing possibility Ref. [1] that the physical spectrum of a vector-like gauge field theory exhibits an enhanced global symmetry near a chiral phase transition. A transition from the Goldstone phase to the symmetric phase is expected as the number of fermions $N_{f}$ is increased to some critical value. Various investigations have suggested that a parity-doubled spectrum develops as the critical value is approached. Using an effective Lagrangian as a guide one observes that a parity doubling is associated with the appearance of an enhanced global symmetry in the spectrum of the theory. If such a near-critical theory describes symmetry breaking in the electroweak theory, the additional symmetry suppresses the contribution of the parity doubled sector to the $S$ parameter.
\end{abstract}

*Electronic address : francesco.sannino@yale.edu 


\section{INTRODUCTION}

Gauge field theories exhibit many different patterns of infrared behavior. Indeed for a vector-like theory such as QCD, it is known that for low values of $N_{f}$, the theory confines and chiral symmetry breaking occurs. On the other hand, for large $N_{f}$ the theory loses asymptotic freedom. In between, there is a conformal window where the theory does not confine, chiral symmetry is restored, and the theory acquires a long range conformal symmetry. It has been proposed that for an $S U(N)$ gauge theory, there is a transition from the confining, chirally broken theory to the chirally symmetric theory at $N_{f} \approx 4 N$ [2, 3]. Recent lattice simulations, however, seem to indicate [4] that the amount of chiral symmetry breaking decreases substantially (for $N=3$ ) when $N_{f}$ is only about 4 .

Assuming that a single transition takes place at some critical value of $N_{f}$, we can ask questions about the spectrum of the theory near the transition. In Reference [5], it was argued by studying Weinberg spectral function sum rules that for near-critical theories parity partners become more degenerate than in QCD-like theories. This leads naturally to the idea that parity doublets might form as chiral symmetry is being restored. Lattice studies also indicate such a possibility [4].

Here I review the ideas presented in Ref. [1] where it was observed using an effectiveLagrangian as a guide, that the formation of degenerate parity partners is associated with the appearance of an enhanced global symmetry in the spectrum of states. This new symmetry could play a key role in describing a possible strong electroweak Higgs sector. Whether the new symmetry can be shown to emerge dynamically from an underlying gauge theory with $N_{f}$ near a critical value remains an open question.

First, in Section II, the appearance of enhanced global symmetry is discussed. Confinement is assumed and the symmetry of the underlying gauge theory, $S U_{L}\left(N_{f}\right) \times S U_{R}\left(N_{f}\right)$, is built into an effective Lagrangian describing the physical states of the theory. Parity invariance is imposed and the usual pattern of chiral symmetry breaking $\left(S U_{L}\left(N_{f}\right) \times S U_{R}\left(N_{f}\right) \rightarrow\right.$ $\left.S U_{V}\left(N_{f}\right)\right)$ is assumed. The $N_{f}^{2}-1$ Goldstone bosons appear together with scalar chiral partners. We augment the spectrum with a set of vector fields for both the $S U_{L}\left(N_{f}\right)$ and $S U_{R}\left(N_{f}\right)$ symmetry groups. The Lagrangian thus takes the form of a linear sigma model coupled to vectors.

Analyzing the spectrum one recognizes that there is a particular choice of the parameters that allows for a degenerate vector and axial-vector, while enlarging the global symmetry to include an additional (unbroken) $S U_{L}\left(N_{f}\right) \times S U_{R}\left(N_{f}\right)$. This happens as the spectrum of the theory splits into two sectors with one displaying the additional symmetry. I then 
review the arguments (see Ref. [5]) that an underlying near-critical $S U(N)$ gauge theory might naturally lead to a more degenerate vector-axial spectrum than in QCD, and to an enhanced symmetry. Finally we note that even a discrete additional symmetry, $Z_{2 L} \times Z_{2 R}$, of the effective theory is adequate to insure the mass degeneracy of the vector and axial vector.

The possible appearance of an additional, continuous symmetry was considered by Casalbuoni et al in Refs. [6.7].

Finally in Section 【II the electroweak gauge group is embedded within the global symmetry group. As observed in Ref. [1] the enhanced symmetry of the strongly interacting sector, which now provides electroweak symmetry breaking, plays an important role. The additional symmetry operates as a partial custodial symmetry for the electroweak $S$ parameter, in the sense that the parity doubled part of the strong sector, by itself, makes no contribution to $S$.

\section{EFFECTIVE LAGRANGIAN FOR $S U_{L}\left(N_{F}\right) \times S U_{R}\left(N_{F}\right)$ GLOBAL SYMMETRY}

To discuss the possible appearance of enhanced symmetry some description of the spectrum is needed. It is helpful to use an effective Lagrangian possessing $S U_{L}\left(N_{f}\right) \times S U_{R}\left(N_{f}\right)$ symmetry, the global invariance of the underlying gauge theory. Chiral symmetry is broken according to the standard pattern $S U_{L}\left(N_{f}\right) \times S U_{R}\left(N_{f}\right) \rightarrow S U_{V}\left(N_{f}\right)$. The $N_{f}^{2}-1$ Goldstone bosons are encoded in the $N_{f} \times N_{f}$ real traceless matrix $\Phi_{j}^{i}$ with $i, j=1, \ldots, N_{f}$. The complex matrix $M=S+i \Phi$ describes both the Goldstone bosons as well as associated scalar

partners $S$. It transforms linearly under a chiral rotation of the type $M \rightarrow u_{L} M u_{R}^{\dagger}$ with $u_{L / R}$ in $S U_{L / R}\left(N_{f}\right)$.

To augment the massive spectrum one introduces vector and axial vector fields following a method outlined in Ref. [9]. One first formally gauge the global chiral group introducing the covariant derivative

$$
D^{\mu} M=\partial^{\mu} M-i \tilde{g} A_{L}^{\mu} M+i \tilde{g} M A_{R}^{\mu}
$$

where $A_{L / R}^{\mu}=A_{L / R}^{\mu, a} T^{a}$ and $T^{a}$ are the generators of $S U\left(N_{f}\right)$, with $a=1, \ldots, N_{f}^{2}-1$ and $\operatorname{Tr}\left[T^{a} T^{b}\right]=\frac{1}{2} \delta^{a b}$. Under a chiral transformation

$$
A_{L / R}^{\mu}=u_{L / R} A_{L}^{\mu} u_{L / R}^{\dagger}-\frac{i}{\tilde{g}} \partial^{\mu} u_{L / R} u_{L / R}^{\dagger} .
$$

The effective Lagrangian needs only to be invariant under global chiral transformations. Including terms only up to mass dimension four, it may be written in the form 


$$
\begin{aligned}
L & =\frac{1}{2} \operatorname{Tr}\left[D_{\mu} M D^{\mu} M^{\dagger}\right]+m^{2} \operatorname{Tr}\left[A_{L \mu} A_{L}^{\mu}+A_{R \mu} A_{R}^{\mu}\right]+h \operatorname{Tr}\left[A_{L \mu} M A_{R}^{\mu} M^{\dagger}\right] \\
& +r \operatorname{Tr}\left[A_{L \mu} A_{L}^{\mu} M M^{\dagger}+A_{R \mu} A_{R}^{\mu} M^{\dagger} M\right]+i \frac{s}{2} \operatorname{Tr}\left[A_{L \mu}\left(M D^{\mu} M^{\dagger}-D^{\mu} M M^{\dagger}\right)\right. \\
& \left.+A_{R \mu}\left(M^{\dagger} D^{\mu} M-D^{\mu} M^{\dagger} M\right)\right] .
\end{aligned}
$$

The parameters $h, r$ and $s$ are dimensionless real parameters, while $m^{2}$ is a common mass term. To this, we may add a kinetic term for the vector fields

$$
L_{\text {Kin }}=-\frac{1}{2} \operatorname{Tr}\left[F_{L \mu \nu} F_{L}^{\mu \nu}+F_{R \mu \nu} F_{R}^{\mu \nu}\right]
$$

where $F_{L / R}^{\mu \nu}=\partial^{\mu} A_{L / R}^{\nu}-\partial^{\nu} A_{L / R}^{\mu}-i \tilde{g}\left[A_{L / R}^{\mu}, A_{L / R}^{\nu}\right]$ along with vector-interaction terms respecting only the global symmetry. Finally, one may add the double trace term, $\operatorname{Tr}\left[M M^{\dagger}\right] \operatorname{Tr}\left[A_{L}^{2}+A_{R}^{2}\right]$ at the dimension-four level. To arrange for symmetry breaking, a potential $V\left(M, M^{\dagger}\right)$ must be added. When the effective Lagrangian is extended to the dimension-six level and higher, many new operators enter. Parity is also a symmetry. It is worth noting that a Lagrangian of this type has been used to describe the low-lying QCD resonances and interactions 10$]$.

The scalar vacuum expectation value is $v$ and the new vector fields are

$$
V=\frac{A_{L}+A_{R}}{\sqrt{2}}, \quad A=\frac{A_{L}-A_{R}}{\sqrt{2}}
$$

The vector-axial vector mass difference is given by

$$
M_{A}^{2}-M_{V}^{2}=v^{2}\left[\tilde{g}^{2}-2 \tilde{g} s-h\right]
$$

In QCD this difference is known experimentally to be positive, a fact that can be understood by examining the Weinberg spectral function sum rules (see Ref. [11] and references therein). The effective Lagrangian description is of course unrestrictive. Depending on the values of the $\tilde{g}, s$ and $h$ parameters, one can have a degenerate or even inverted mass spectrum.

What kind of underlying gauge theory might provide a degenerate or inverted spectrum? Clearly, it has to be different from QCD, allowing for a modification of the spectral function sum rules. In Reference [5], an $S U(N)$ gauge theory (with $N>2$ ) and $N_{f}$ flavors was considered. If $N_{f}$ is large enough but below $11 N / 2$, an infrared fixed point of the gauge coupling $\alpha_{*}$ exists, determined by the first two terms in the $\beta$ function. For $N_{f}$ near $11 N / 2$, $\alpha_{*}$ is small and the global symmetry group remains unbroken. For small $N_{f}$, on the other hand, the chiral symmetry group $S U_{L}\left(N_{f}\right) \times S U_{R}\left(N_{f}\right)$ breaks to its diagonal subgroup. One possibility is that the transition out of the broken phase takes place at a relatively large value of $N_{f} / N(\approx 4)$, corresponding to a relatively weak infrared fixed point [2, 3]. An alternate 
possibility is that the transition takes place in the strong coupling regime, corresponding to a small value of $N_{f} / N$ [4. The larger value emerges from the renormalization group improved gap equation, as well as from instanton effects [12], and saturates a recently conjectured upper limit [13]. It corresponds to the perturbative infrared fixed point $\alpha_{*}$ reaching a certain critical value $\alpha_{c}$. A similar result has also been obtained by using a suitable effective Lagrangian [3].

In Reference [5] the spectrum of states in the broken phase near a large- $N_{f} / N$ transition was investigated using the spectral function sum rules. It was shown that the ordering pattern for vector-axial hadronic states need not be the same as in QCD-like theories (small $\left.N_{f} / N\right)$. The crucial ingredient is that these theories contain an extended "conformal region" extending from roughly $2 \pi F_{\pi}$ to the scale $\Lambda$ where asymptotic freedom sets in. In this region, the coupling remains close to an approximate infrared fixed point and the theory has an approximate long range conformal symmetry. It was argued that this leads to a reduced vector-axial mass splitting, compared to QCD-like theories. This suggests the interesting possibility that parity doublets begin to form as chiral symmetry is being restored. That is, the vector-axial mass ratio approaches unity as the masses decrease relative to $\Lambda$. Lattice results seem to provide supporting evidence for such a possibility [4], although at smaller values of $N_{f} / N$.

If a parity doubled spectrum does appear, it is natural to expect it to be associated with some new global symmetry. While it is hard to demonstrated the appearance of a new global symmetry using the underlying degrees of freedom, one can explore aspects of parity doubling at the effective Lagrangian level. Returning to this description, we note that vector-axial parity doubling corresponds to the parameter choice (see Eq. (2.6)),

$$
\tilde{g}^{2}=2 \tilde{g} s+h
$$

This condition does not yet reveal an additional symmetry and therefore there is no reason to expect parity degeneracy to be stable in the presence of quantum corrections and the many higher dimensional operators that can be added to the effective Lagrangian in Eq. (2.3).

However, for the special choice $s=\tilde{g}, r=\tilde{g}^{2} / 2$ and $h=-\tilde{g}^{2}$, the effective Lagrangian acquires a new continuous global symmetry that protects the vector-axial mass difference. The effective Lagrangian at the dimension-four level takes the simple form

$$
L=\frac{1}{2} \operatorname{Tr}\left[\partial_{\mu} M \partial^{\mu} M^{\dagger}\right]+m^{2} \operatorname{Tr}\left[A_{L \mu} A_{L}^{\mu}+A_{R \mu} A_{R}^{\mu}\right]
$$

along with vector kinetic and interaction terms, the interaction term Eq. (III), and the symmetry breaking potential $V\left(M, M^{\dagger}\right)$. The theory now has two sectors, with the vector and axial vector having their own unbroken global $S U_{L}\left(N_{f}\right) \times S U_{R}\left(N_{f}\right)$. The two 
sectors interact only through the product of singlet operators. The full global symmetry is $\left[S U_{L}\left(N_{f}\right) \times S U_{R}\left(N_{f}\right)\right]^{2} \times U_{V}(1)$ spontaneously broken to $S U_{V}\left(N_{f}\right) \times U_{V}(1) \times$ $\left[S U_{L}\left(N_{f}\right) \times S U_{R}\left(N_{f}\right)\right]$. The vector and axial vector become stable due to the emergence of a new conservation law. This enhanced symmetry would become exact only in the chiral limit. For finite but small (relative to $\Lambda$ ) values of the mass scales in Eq. (2.8), there are additional, smaller terms giving smaller mass splittings and small width-to-mass ratios.

It is of course a simple observation that a new symmetry and conservation law emerge if a theory is split into two sectors by setting certain combinations of parameters to zero. But here we were led to this possibility by looking for a symmetry basis for the parity doubling that has been hinted at by analyses of the underlying gauge theory.

It is worth mentioning that along with the additional global symmetry $S U_{L}\left(N_{f}\right) \times$ $S U_{R}\left(N_{f}\right)$, the effective Lagrangian Eq. (2.8) possesses a discrete $Z_{2 L} \times Z_{2 R}$ symmetry. Under $Z_{2 L} \times Z_{2 R}$ the vector fields transform according to $A_{L} \rightarrow z_{L} A_{L}, A_{R} \rightarrow z_{R} A_{R}$ with $z_{L / R}=1,-1$ and $z_{L / R} \in Z_{2 L / R}$. Actually, the discrete symmetry alone is enough to insure vector-axial mass degeneracy and stability against decay. In that case, additional interaction terms, such as the single trace term $r \operatorname{Tr}\left[A_{\mu L} A_{L}^{\mu} M M^{\dagger}+A_{\mu R} A_{R}^{\mu} M^{\dagger} M\right]$ are allowed, but degeneracy and stability are still insured.

\section{STRONGLY INTERACTING ELECTROWEAK SECTOR}

To discuss the consequences of enhanced symmetry for a strong symmetry breaking sector of the standard electroweak theory one embeds the $S U_{L}(2) \times U_{Y}(1)$ gauge symmetry in the global $S U_{L}\left(N_{f}\right) \times S U_{R}\left(N_{f}\right)$ chiral group. For simplicity I restrict attention to the $S U_{L}(2) \times S U_{R}(2)$ subgroup of the full global group. The electroweak gauge transformation then takes the form $M \rightarrow u_{W} M u_{Y}^{\dagger}$. $M$ is now a $2 \times 2$ matrix which can be written as $M=\frac{1}{\sqrt{2}}[\sigma+i \vec{\tau} \cdot \vec{\pi}]$, where $u_{W}=u_{L}=\exp \left(\frac{i}{2} \epsilon^{a} \tau^{a}\right)$ with $\tau^{a}$ the Pauli matrices, and where $u_{Y}=\exp \left(\frac{i}{2} \epsilon_{0} \tau^{3}\right)$. The weak vector boson fields transform as

$$
W^{\mu} \rightarrow u_{L} W^{\mu} u_{L}^{\dagger}-\frac{i}{g} \partial^{\mu} u_{L} u_{L}^{\dagger}, \quad B^{\mu} \rightarrow u_{Y} B^{\mu} u_{Y}^{\dagger}-\frac{i}{g^{\prime}} \partial^{\mu} u_{Y} u_{Y}^{\dagger}
$$

where $g$ and $g^{\prime}$ are the standard electroweak coupling constants, $W_{\mu}=W_{\mu}^{a} \frac{\tau^{a}}{2}$ and $B_{\mu}=B_{\mu} \frac{\tau^{3}}{2}$.

A convenient method of coupling the electroweak gauge fields to the globally invariant effective Lagrangian in Section $\mathbb{I}$ is to introduce a covariant derivative, which includes the $W$ and $B$ fields as well as the strong vector and axial-vector fields,

$$
\mathrm{D}^{\mu} M=\partial^{\mu} M-i g W^{\mu} M+i g^{\prime} M B^{\mu}-i \tilde{g} c C_{L}^{\mu} M+i \tilde{g} c^{\prime} M C_{R}^{\mu}
$$


where we have defined the new vector fields $C_{L}^{\mu}=A_{L}^{\mu}-\frac{g}{\tilde{g}} W^{\mu}, C_{R}^{\mu}=A_{R}^{\mu}-\frac{g^{\prime}}{\tilde{g}} B^{\mu}$ and where $c$ and $c^{\prime}$ are arbitrary real constants (for details see Ref. [1]). By requiring invariance under the parity operation exchanging the labels $L \leftrightarrow R$ we have the extra condition $c=c^{\prime}$.

The effective Lagrangian, constructed to be invariant under a local $S U_{L}(2) \times U_{Y}(1)$ as well as $C P$ is through dimension four,

$$
\begin{aligned}
L & =\frac{1}{2} \operatorname{Tr}\left[\mathrm{D}_{\mu} M \mathrm{D}^{\mu} M^{\dagger}\right]+m^{2} \operatorname{Tr}\left[C_{L \mu} C_{L}^{\mu}+C_{R \mu} C_{R}^{\mu}\right]+h \operatorname{Tr}\left[C_{L \mu} M C_{R}^{\mu} M^{\dagger}\right] \\
& +r \operatorname{Tr}\left[C_{L \mu} C_{L}^{\mu} M M^{\dagger}+C_{R \mu} C_{R}^{\mu} M^{\dagger} M\right]+i \frac{s}{2} \operatorname{Tr}\left[C_{L \mu}\left(M \mathrm{D}^{\mu} M^{\dagger}-\mathrm{D}^{\mu} M M^{\dagger}\right)\right. \\
& \left.+C_{R \mu}\left(M^{\dagger} \mathrm{D}^{\mu} M-\mathrm{D}^{\mu} M^{\dagger} M\right)\right] .
\end{aligned}
$$

To this we add a kinetic term

$$
L_{\text {Kin }}=-\frac{1}{2} \operatorname{Tr}\left[F_{L \mu \nu} F_{L}^{\mu \nu}+F_{R \mu \nu} F_{R}^{\mu \nu}\right]-\frac{1}{2} \operatorname{Tr}\left[W_{\mu \nu} W^{\mu \nu}\right]-\frac{1}{2} \operatorname{Tr}\left[B_{\mu \nu} B^{\mu \nu}\right],
$$

where $W_{\mu \nu}=\partial_{\mu} W_{\nu}-\partial_{\nu} W_{\mu}-i g\left[W_{\mu}, W_{\nu}\right]$ and $B_{\mu \nu}=\partial_{\mu} B_{\nu}-\partial_{\nu} B_{\mu}$. One should still add other interaction terms involving the $C_{L / R}$ fields, the interaction term $\operatorname{Tr}\left[M M^{\dagger}\right] \operatorname{Tr}\left[C_{L}^{2}+C_{R}^{2}\right]$ and a symmetry breaking potential.

The extension of this effective Lagrangian to the relevant case of the larger symmetry group $S U_{L}\left(N_{f}\right) \times S U_{R}\left(N_{f}\right)$ with $N_{f}>2$, is straightforward.

The vector and axial vector masses computed in Ref. [1], $M_{V}^{2}$ and $M_{A}^{2}$, are arbitrary, depending on the choice of parameters, although generically we expect them and the scalar masses to be of order $4 \pi^{2} v^{2}$.

Expanding the Lagrangian up to quadratic terms in the fields one observes the presence of weak mixing terms providing a contribution from physics beyond the standard model to the oblique electroweak corrections. (One can find the actual computations and more explicit formulae in Ref. [1]). These may be described by the $S, T$, and $U$ parameters, but the last two vanish in the present model because there is no breaking of weak isospin in the strong sector. The $S$ parameter receives contributions from all the physics beyond the standard model, including, in the model being used here, loops of pseudo-Goldstone bosons (PGB's), the strongly interacting massive scalars, and the vector and axial vector. The direct, vector-dominance contribution of the vector and axial vector is

$$
S_{\text {vect-dom }}=\frac{8 \pi}{\tilde{g}^{2}}\left[\frac{M_{A}^{2}(1-\chi)^{2}}{M_{Z}^{2}-M_{A}^{2}}-\frac{M_{V}^{2}}{M_{Z}^{2}-M_{V}^{2}}\right] \approx \frac{8 \pi}{\tilde{g}^{2}}\left[1-(1-\chi)^{2}\right] .
$$

with $\chi=\frac{v^{2}}{2 M_{A}^{2}} \tilde{g}[\tilde{g} c-s]$. Clearly, this contribution to the $S$ parameter can take on any value depending on the choice of parameters. Its typical order of magnitude, with the strong coupling estimate $\tilde{g}^{2} \approx 4 \pi^{2}$, is expected to be $O(1)$. 
The parameter choice $s=\tilde{g} c$ and $h=-\tilde{g}^{2} c^{2}$ gives $\chi=0$, leads to the degeneracy of the vector and axial vector and the vanishing of $S_{v e c t-d o m}$. The further choice $r=\frac{\tilde{g}^{2} c^{2}}{2}$ leads to the collapse of the general effective Lagrangian into the simple form

$$
L=\frac{1}{2} \operatorname{Tr}\left[D_{\mu} M D^{\mu} M^{\dagger}\right]+m^{2} \operatorname{Tr}\left[C_{L \mu} C_{L}^{\mu}+C_{R \mu} C_{R}^{\mu}\right]
$$

along with the kinetic terms of Eq. (3.4), interactions among the $C_{L / R}^{\mu}$ fields and a symmetry breaking potential. Here, $D M=\partial M-i g W M+i g^{\prime} M B$ is the standard electroweak covariant derivative.

The strongly interacting sector has split into two subsectors, communicating only through the electroweak interactions. One subsector consists of the Goldstone bosons together with their massive scalar partners. The other consists of the degenerate vector and axial vector described by the $A_{L / R}^{\mu}$ fields. In the absence of electroweak interactions, there is an enhanced symmetry $\left[S U_{L}(2) \times S U_{R}(2)\right] \times\left[S U_{L}(2) \times S U_{R}(2)\right]$, breaking spontaneously to $S U_{V}(2) \times$ $\left[S U_{L}(2) \times S U_{R}(2)\right]$. The electroweak interactions explicitly break the enhanced symmetry to $S U_{L}(2) \times U_{Y}(1)$. All of this may be generalized to $N_{f}>2$, necessary to yield a near-critical theory.

The additional symmetry has an important effect on the $S$ parameter, suppressing contributions that are typically large in QCD-like theories.

Finally, it could be that only a lesser, discrete symmetry emerges in the physical spectrum. Even this would be sufficient to insure vector-axial degeneracy and the vanishing of the vector dominance contribution to the $S$ parameter.

\section{CONCLUSIONS}

To explore some features that might arise in a strongly coupled gauge theory when the number of fermions $N_{f}$ is near a critical value for the transition to chiral symmetry an effective Lagrangian approach was used.

The spectrum was taken to consist of a set of Goldstone particles, associated massive scalars, and a set of massive vectors and axial vectors. It was observed that parity doubling is associated with the appearance of an enhanced global symmetry, consisting of the spontaneously broken chiral symmetry of the underlying theory $\left(S U_{L}\left(N_{f}\right) \times S U_{R}\left(N_{f}\right)\right)$ together with an additional, unbroken symmetry, either continuous or discrete. The additional symmetry leads to the degeneracy of the vector and axial vector, and to their stability with respect to decay into the Goldstone bosons. 
Despite the hints in Refs. [5,4] it has not been established that an underlying gauge theory leads to these enhanced symmetries as $N_{f}$ approaches a critical value for the chiral transition. If it is to happen an interesting interplay between confinement and chiral symmetry breaking would have to develop at the transition.

Finally by electroweak gauging a subgroup of the chiral flavor group it was shown that the enhanced symmetry provides a partial custodial symmetry for the $S$ parameter, in that there is no contribution from the parity-doubled sector by itself.

\section{ACKNOWLEDGMENTS}

I am happy to thank Thomas Appelquist and Paulo Sergio Rodrigues Da Silva for sharing the work on which this talk is based. The work of F.S. has been partially supported by the US DOE under contract DE-FG-02-92ER-40704.

[1] T. Appelquist, P.S. Rodrigues da Silva and F. Sannino, Report Numbers: YCTP-P14-99, hep-ph/9906555. To be published on Phys. Rev. D.

[2] T. Appelquist, J. Terning and L.C.R. Wijewardhana, Phys. Rev. Lett. 77, 1214 (1996).

[3] F. Sannino and J. Schechter, Phys. Rev. D60, 056004, (1999).

[4] R.D. Mawhinney, Nucl. Phys. Proc. Suppl. A 60, 306 (1998), Report Numbers: CU-TP-802, hep-lat/9705030; D. Chen, R.D. Mawhinney, Nucl. Phys. Proc. Suppl. 53, 216 (1997).

[5] T. Appelquist and F. Sannino, Phys. Rev. D59, 067702 (1999).

[6] R. Casalbuoni, S. De Curtis, D. Dominici, F. Feruglio and R. Gatto, Int. J. Mod. Phys. A4, 1065 (1989); R. Casalbuoni, A. Deandrea, S. De Curtis, D. Dominici, F. Feruglio, R. Gatto and M. Grazzini, Phys. Lett. B349, 533 (1995); R. Casalbuoni, A. Deandrea, S. De Curtis, D. Dominici, R. Gatto and M. Grazzini, Phys. Rev. D53, 5201 (1996).

[7] R. Casalbuoni, S. De Curtis, D. Dominici and M. Grazzini, Phys. Lett. B388, 112 (1996); Phys. Rev D56, 5731 (1997).

[8] M. Bando, T. Kugo and K. Yamawaki, Phys. Rept. 164, 217 (1988).

[9] Ö. Kaymakcalan and J. Schechter, Phys. Rev. D31, 1109 (1985). 
[10] F. Sannino and J. Schechter, Phys. Rev. D52, 96 (1995); M. Harada, F. Sannino and J. Schechter, Phys. Rev. D54, 1991 (1996); Phys. Rev. Lett. 78, 1603 (1997); D. Black, A.H. Fariborz, F. Sannino and J. Schechter, Phys. Rev. D58, 54012 (1998).

[11] C. Bernard, A. Duncan, J. LoSecco and S. Weinberg, Phys. Rev. D12, 792 (1975).

[12] T. Appelquist and S. Selipsky, Phys. Lett. B400, 364 (1997); M. Velkovsky and E. Shuryak, Phys. Lett. B437, 398 (1998).

[13] T. Appelquist, A. Cohen and M. Shmaltz, Report Numbers: Phys. Rev. D60, 045003 (1999).

[14] M. E. Peskin and T. Takeuchi, Phys. Rev. Lett. 65, 964 (1990); Phys. Rev. D46, 381 (1992).

[15] A. Longhitano, Phys. Rev. D22, 1166 (1980); Nucl. Phys. B188, 118 (1981).

[16] T. Appelquist and J. Terning, Phys. Lett. B315, 139 (1993); S. Fleming and I. Maksymyk, Phys. Rev. D53, 2781 (1996).

[17] T. Appelquist, J. Terning, and L.C.W. Wijewardhana, Phys. Rev. Lett. 79, 2767 (1997).

[18] S. Hands, J. Kogut, M. Lombardo and S. Morrison, Report Number: hep-lat/9902034.

[19] M. E. Peskin, Nucl. Phys. B175, 197 (1980), J. Preskill, Nucl. Phys. B177, 21 (1981). 